\title{
Etat nutritionnel des enfants réfugiés : Synthèses des cas des Libériens en Guinée et en Côte-d'Ivoire et des Somaliens en Ethiopie
}

\author{
Kuakuvi Gbenyon
}

\section{Résumé}

Les réfugiés constituent une catégorie assez particulière de la population., pour laquelle se pose un problème de définition qui n'est pas abordé par les différentes études qui lui sont consacrées.

Lorsqu'on considère l'état nutritionnel des enfants réfugiés âgés de 0 à 5 ans, celui-ci est souvent des plus précaires. Les synthèses faites montrent qu'un des facteurs qui favoriserait le maintien d'un état nutritionnel satisfaisant pour les populations, après les troubles sociaux, est l'intégration de la population réfugiée dans la population autochtone sans construction de camps de réfugiés.

Ainsi, les enfants réfugiés libériens en Guinée et en Côte-d'Ivoire en 1990-1992, intégrés dans la population autochtone, avaient un état nutritionnel satisfaisant, identique à celui de la population autochtone, tandis que ceux de la Somalie réfugiés en Ethiopie en 1988-1989, accueillis dans des camps de réfugiés, avaient un mauvais état nutritionnel comparativement à celui de la population autochtone.

L'étude montre que les stratégies efficaces de prise en charge des réfugiés et d'amélioration de leur état nutritionnel, surtout celui des enfants, pourraient comporter:

- La distribution régulière et complète de rations alimentaires comportant des aliments riches en vitamine $C$, et

- l'extension des programmes d'alimentation d'appoint et de thérapeutique pour une meilleure couverture de la population réfugiée.

\section{Summary}

Refugees constitute a rather special category of the population. For this sub-group of the entire population, a problem of definition is raised which unfortunately has not been addressed by the different studies devoted to the refugee population.

When we consider the nutritional status of refugee children aged between 0 and 5 years, it appears that it is of the most precarious ones. The syntheses made show that one of the factors which would favour the maintenance of a satisfactory nutritional status for the populations, following social unrest, is the integration of the refugee population into the indigenous population without the construction of refugee camps.

Thus, liberian refugee children in Guinea and in Côte d'Ivoire in 1990-1992, who are integrated into the indigenous population, had a satisfactory nutritional status identical to that of the indigenous population, whereas those somali refugees in Ethiopia in 1988-1989, hosted in refugee camps, had a poor nutritional status when compared to that of the indigenous population.

The study shows that efficient strategies of refugee management and improvement of their nutritional status, especially that of children, might comprise:

- the regular and complete distribution of food ratios comprising foods rich in vitamin $C$ and,

- the extension of makeshift food and therapy programmes for a better coverage of the refugee population.

\section{Introduction}


Il est généralement admis que l'anthropométrie est l'outil le plus commode d'évaluation de l'état nutritionnel des enfants, bien que les variations des courbes de croissance tiennent à diverses raisons (maladies par exemple) et non pas seulement à la nutrition. Toutefois, on peut remarquer que les propositions sur l'utilisation des données relatives au poids et à la taille des enfants sont largement acceptées. Dans cette optique, nous nous proposons de faire la synthèse de trois cas d'utilisation des indices poids-taille dans l'évaluation de l'état nutritionnel des enfants en Afrique, dans un contexte de troubles sociaux ou de guerre civile. Nous tenterons ainsi d'apprécier l'effet des troubles sociaux sur l'état nutritionnel des enfants.

Nous examinerons le cas des enfants réfugiés Libériens en Côte-d'Ivoire et en Guinée (cas de malnutrition satisfaisant), et celui des Somaliens en Ethiopie (cas de malnutrition grave). II s'agit donc de l'étude d'un sous-groupe particulier, celui des enfants réfugiés, dont l'état de santé, la morbidité et la mortalité sont généralement mal connus.

Auparavant, nous apprécierons la pertinence de l'utilisation de la population de référence du United States National Center for Health Statistics (NCHS) pour l'évaluation de l'état nutritionnel des enfants, étant donné que cet usage soulève quelques questions méthodologiques auxquelles il convient d'apporter des réponses. Nous parlerons aussi de la fiabilité des indices, de l'échantillonnage et du problème de définition des réfugiés.

\section{Considérations méthodologiques}

Utilisation de la population de référence du NCHS

Les études de cas dont nous allons faire état se réfèrent toutes à la norme du NCHS. Un tel usage est-il justifié ?

Les débats se sont poursuivis ces dernières années pour savoir s'il est nécessaire et adéquat d'utiliser une norme internationale pour évaluer l'état nutritionnel des enfants.

En fait, la justification de l'utilisation d'un standard a été apporté par certains auteurs (Habicht et al., 1974) lorsqu'ils ont démontré que dans diverses populations, l'effet des différences ethniques sur la croissance des enfants est peu important comparativement aux effets de l'environnement. II est admis qu'il peut y avoir quelques différences ethniques entre les groupes de populations, comme c'est le cas des différences génétiques entre individus, mais ces différences ne sont pas significatives pour invalider l'utilisation à grande échelle de la population du NCHS à la fois comme population de référence et comme standard. Ce point de vue a été appuyé dans un rapport conjoint de la FAO, de l'OMS et des Nations-Unies (WHO, 1985).

Dans les pays où des standards ont été conçus (Colombie, Mexique et Pérou), on a remarqué que ces standards différaient peu de la norme du NCHS. En outre, le développement de normes nationales statistiquement valides est souvent coûteux par rapport aux avantages obtenus et se heurte à des problèmes logistiques, notamment lorsque l'effectif de la population à étudier est grand si bien que la mise au point de normes locale ou nationale ne semble pas être un problème prioritaire. D'un autre côté, un problème demeure, celui de la fiabilité des indices utilisés pour mesurer l'état nutritionnel des enfants et la caractérisation de ce dernier.

\section{Fiabilité des indices et caractérisation de la malnutrition}

Les variables généralement prises en compte dans l'évaluation de l'état nutritionnel des enfants sont l'âge, le poids et la taille. La mesure de ces variables pose des problèmes de fiabilité et de précision que nous nous contenterons de rappeler. Ceci dit, le poids, pris isolément, n'a pas grande signification à moins qu'il ne soit mis en relation avec l'âge ou la taille. Aussi, les indices requis pour l'analyse sont une combinaison de ces variables.

Pour les enfants, l'utilisation de deux indices, le "poids selon la taille" et la "taille selon l'âge" sont utiles pour la plupart des besoins. Dans certains cas, l'indice "poids selon l'âge"peut être plus utile pour donner une vue générale sur la distribution des problèmes nutritionnels dans un pays. 
Les trois études de cas dont nous allons faire la synthèse ont surtout utilisé l'indice du "poids selon la taille", qui est particulièrement important pour la description de l'état actuel de santé des enfants, et pour l'évaluation du nombre d'enfants sous-alimentés. D'une manière générale, cet indice est également utilisé pour identifier des groupes-cibles ou des zones géographiques nécessitant des actions prioritaires. Son équivalent est la mesure de la "circonférence du bras selon la taille".

En ce qui concerne la caractérisation de la malnutrition, étant donné que l'amaigrissement et le retard de croissance se réfèrent à deux processus biologiques différents de malnutrition, il faut noter que l'indicateur du "poids selon la taille" se réfère à l'amaigrissement, tandis que l'indicateur de la "taille selon l'âge" a trait au retard de croissance (WHO, 1986, p.938).

\section{Echantillonnage}

La taille de l'échantillon tout comme le nombre de mesures à effectuer dépendent des ressources financières disponibles. Ainsi, les tailles d'échantillon utilisées dans les trois études de cas ainsi que d'autres caractéristiques se présentent comme suit (tableau 1):

Etant donné que les groupes-cibles d'enfants examinés sont variables, tout comme le milieu d'enquête (urbain ou rural), nous ne pourrons pas rapprocher les cas somalien et libérien et faire des comparaisons valides; aussi procéderons-nous à une synthèse de chaque cas en veillant à faire ressortir les points communs.

Par contre, la méthode de sondage mise en oeuvre pour les trois études est celle du sondage en grappes avec probabilités proportionnelles à la taille des populations.

Par ailleurs nous évoquerons un problème commun à toutes les enquêtes auprès des réfugiés: celui de la définition du réfugié. Nous ne ferons que le soulever, puisque les cas que nous avons recueillis ne donnent aucune indication sur la définition de la population réfugiée adoptée; ce qui n'autorise pas non plus une comparaison valide.

\section{Décompte des réfugiés : un problème de définition}

Dans la plupart des programmes d'assistance administrés par le Haut commissariat des NationsUnies pour les réfugiés (UNHCR), le terme "population réfugiée" peut renvoyer à l'un des cinq groupes qui sont les suivants (UNHCR, 1993, p.7) :

- le nombre de réfugiés enregistrés par le gouvernement du pays hôte (problème de la mauvaise conception du terme réfugié, double décompte, manipulation, etc.);

- le nombre de personnes prises en charge ou reconnues par I'UNHCR;

- le nombre de personnes supposées recevoir une assistance;

- le nombre de personnes présentes dans un camp de réfugiés ou dans une zone correspondante (certaines personnes peuvent ne pas être des réfugiés);

- enfin le nombre de personnes bénéficiant dans le présent d'une assistance.

- En réalité, les besoins en données chiffrées d'un fonctionnaire du HCR (agent chargé de la protection ou de l'information publique, etc.) peuvent différer de ceux d'un nutritionniste ou d'un spécialiste de la santé publique. Aussi, il peut être plus réaliste de penser en termes de différents groupes-cibles de réfugiés plutôt que d'une population unique de réfugiés.

Face à la diversité des définitions qui peut biaiser les comparaisons internationales, les efforts de collecte des données statistiques devraient partir d'une définition claire et sans équivoque de la population concernée. Ceci nous amène à examiner les objectifs assignés aux évaluations des cas qui nous concernent.

\section{Objectifs des différentes évaluations}

L'utilité d'une évaluation anthropométrique porte sur plusieurs aspects, allant de la planification nationale à l'identification des personnes à risques. Les enquêtes présentées ici ont été menées dans 
le cadre de l'assistance médicale à apporter aux réfugiés. Toutefois, quelques objectifs spécifiques étaient assignés à ces enquêtes.

a). Dans le cas des enfants réfugiés libériens en Côte-d'Ivoire, il s'agissait de déterminer la prévalence de la malnutrition chez les enfants réfugiés et non réfugiés résidant en milieu rural et de comparer l'état nutritionnel de la population réfugiée à celui de la population autochtone (Soro, 1993).

b). En ce qui concerne les enfants réfugiés libériens en Guinée, il s'agissait, outre la comparaison de l'état nutritionnel de la population autochtone à celui des réfugiés :

- d'établir les priorités voulues en matière de secours;

- de déterminer : l'état vaccinal pour la rougeole;

- la présence d'un oedème prétibial (signe de

- Kwashiorkor); la survenue d'épisodes

- diarrhéiques ou d'autres maladies; les signes

- cliniques d'avitaminose A; enfin,

c). pour ce qui est des enfants réfugiés somaliens dans l'Est de l'Ethiopie, les enquêtes effectuées (huit en tout entre septembre 1988 et mai 1989) rentraient dans le cadre de la surveillance nutritionnelle de routine dans les camps de réfugiés.

A quels résultats a-t-on abouti?

\section{Etat nutritionnel des enfants réfugiés libériens et somaliens : principaux résultats}

Cas des enfants Libériens âgés de 6 mois à 5 ans en Côte-d'Ivoire

Les troubles sociaux débouchant sur une guerre civile ont débuté en décembre 1989 au Libéria. Ils ont entraîné d'importants mouvements de réfugiés à destination des pays limitrophes, notamment la Côte-d'Ivoire et la Guinée.

La population totale réfugiée en Côte-d'Ivoire représentait environ 220821 personnes en 1991 (Bocoum, 1991) dont 175000 répartis dans les départements de Danané, de Guiglo et de Tabou. Seuls les villages des zones d'accueil des départements de Danané et de Guiglo ont servi de base de sondage à la présente évaluation ${ }^{0}$. Dans ces deux départements, la population réfugiée vivait dans les villages ivoiriens, "intégrée" aux familles autochtones; il n'y avait donc pas de camps de réfugiés.

Au total, 2019 enfants ont été examinés répartis comme suit :

- A Danané, 509 enfants libériens et 504 enfants ivoiriens;

- A Guiglo, 511 enfants libériens et 495 enfants ivoiriens.

L'indice utilisé pour mesurer la prévalence de la malnutrition chez les enfants était le rapport poids/taille (PT) exprimé en écarts-types (ET). C'est un indice, particulièrement important pour la description de l'état de santé du moment et approprié pour décrire l'état de santé des enfants. Au niveau individuel, la malnutrition aiguë a été définie comme la présence d'un indice PT inférieur à moins deux écarts-types. Au niveau de la population, la prévalence de la malnutrition est la proportion d'enfants ayant un indice PT inférieur à moins deux écarts-types (SORO, 1993, p.6).

Sur cette base, les résultats suivants ont été obtenus :

- Les prévalences observées ont révélé un état nutritionnel identique dans les populations réfugiées et autochtones (Tableau 2).

- La couverture vaccinale contre la rougeole est très faible chez les enfants Libériens $1 \%$ contre $32 \%$ chez les enfants Ivoiriens. 
Rappelons que dans la population de référence (NCHS), par définition, 2,3\% des sujets ont un indice PT <-2 ET. Cependant, cet indice PT exprimé en ET considère l'état nutritionnel d'une population comme :

- Satisfaisant, si la proportion d'enfants malnutris est inférieure à 5\%;

- Précaire, si cette proportion est comprise entre $5 \%$ et $10 \%$;

- Sévère, si elle est comprise entre $10 \%$ et $15 \%$;

- Très sévère à partir de $15 \%$.

- L'étude a par ailleurs montré, lorsqu'on considère la distribution par âge des enfants, une prédominance de la malnutrition chez les enfants de moins de deux ans aussi bien chez les réfugiés que chez les autochtones, à Guiglo, comme à Danané. Une explication plausible est le sevrage difficile pour cette tranche d'âge.

Toutefois, il y a un certain nombre de questions auxquelles l'étude n'apporte pas de réponses :

a). La proximité géographique ou ethnique des réfugiés et des autochtones peut-elle expliquer l'identité des résultats ?

b). Quels ont été les effets, sur le résultat, des programmes alimentaires des organismes humanitaires qui ont oeuvré dans ces départements?

c). Quels ont été les facteurs socio-économiques et culturels favorisants ?

La réponse à ces questions permettrait de mieux comprendre les résultats précédents pour mieux orienter les actions humanitaires dans d'autres régions du pays ou ailleurs.

Qu'en est-il des enfants libériens réfugiés en Guinée ?

\section{Cas des enfants libériens âgés de 6 mois à 5 ans en Guinée}

Ici, l'ensemble des réfugiés (tous âges confondus) se sont d'abord installés dans la région des forêts où ils ont été accueillis par des membres des mêmes groupes ethniques (principalement Gio et Mano) résidant dans cette zone; les autochtones leur ont offert toit et nourriture. Le nombre des réfugiés a cependant dépassé la capacité des villages concernés à satisfaire leurs besoins fondamentaux; aussi des camps ont-ils été installés; les réfugiés de ces camps ont bénéficié d'une aide extérieure importante (WHO, 1991, p.112).

De mai à décembre 1990, le nombre de réfugiés de ces camps est passé de 80000 à 400000 individus.

L'indice utilisé ici, bien que basé sur le poids par rapport à la taille, est légèrement différent du cas précédent. En effet, les résultats anthropométriques ont été calculés à partir des scores centrésréduits et des pourcentages de la médiane de la population de référence du NCHS.

Ainsi la malnutrition aiguë a été définie comme un poids par rapport à la taille inférieur à $80 \%$ de la médiane de la population de référence (ce qui correspond à un score centré réduit poids/taille inférieur à -2 ET du tableau précédent).

Les résultats obtenus ont montré que :

- la prévalence de la malnutrition était la même chez les enfants guinéens et les enfants libériens;

- $0,3 \%$ et $0,5 \%$ des enfants guinéens et libériens respectivement souffraient de la malnutrition grave (poids par rapport à la taille inférieur à $70 \%$ de la médiane de la population de référence) (tableau 3);

- $1 \%$ des enfants montraient un signe du Kwashiorkor; 
- aucun enfant d'un sous-échantillon de 200 enfants examinés ne présentait des signes cliniques d'avitaminose A.

Cas des enfants réfugiés somaliens âgés de moins de 5 ans en Ethiopie

Pendant l'été 1988, jusqu'à 400000 réfugiés venus du nord de la Somalie sont entrés dans des régions isolées de l'Est de l'Ethiopie. Ils ont été répartis dans plusieurs camps :

- I'un situé près du hameau de Hartisheik,

- l'autre à Harshin, à environ $50 \mathrm{~km}$ de Hartisheik, et

- trois camps près d'Aware.

II n'y avait de points d'eau à aucun de ces endroits; l'eau pourrait cependant être transportée par camion depuis la ville de Jijiga, située à $100 \mathrm{~km}$.

Dans le cadre de la surveillance nutritionnelle de routine dans les camps, des enquêtes par sondage ont été conduites à Hartisheik et harshin entre septembre 1988 et mai 1989 par le Fonds "Save The Children" du Royaume-Uni, organisation privée travaillant avec le gouvernement éthiopien et des institutions des Nations-Unies.

La médiane de la population de référence du NCHS a été utilisée comme dans le cas des Libériens en Guinée.

Ici aussi la malnutrition grave a été définie comme correspondant à des valeurs inférieures à $70 \%$ de la médiane de la population de référence NCHS (Tableau 4) et la malnutrition modérée comme correspondant à des valeurs situées entre $70 \%$ et $79 \%$ de la médiane du NCHS.

Ainsi $40 \%$ des enfants de moins de 5 ans ont été jugés atteints de malnutrition modérée ou grave au cours de l'enquête de janvier 1989; de ce fait, ils ont été inscrits pour les programmes d'alimentation d'appoint dans les camps.

II apparaît (tableau 4) que le problème de la malnutrition chez les enfants réfugiés somaliens dans les deux camps de l'est de l'Ethiopie était critique. En effet, le taux de prévalence de la malnutrition chez ces enfants de réfugiés étaient nettement plus élevés que ceux enregistrés au sein des populations des réfugiés du Libéria. La présence d'enfants de moins de 6 mois dans l'échantillon somalien peutelle expliquer ces différences ? II semble que des taux analogues ont été observés dans des populations de réfugiés au sein desquelles a été enregistré un taux élevé de mortalité (Soudan par exemple). Aussi le HCR a-t-il recommandé l'établissement systématique de statistique de mortalité dans les camps de réfugiés. "De telles statistiques sont particulièrement importantes dans les cas où les taux de malnutrition sont élevés, car les décès survenus chez les plus mal nourris peuvent réduire le nombre et la prévalence des survivants mal nourris et compliquer ainsi l'interprétation des résultats des enquêtes nutritionnelles conduites par les organismes de secours" (WHO, ibid, p. 95.).

Quelle synthèse pouvons-nous faire des résultats précédents?

\section{Discussion des résultats et synthèse}

En guise de synthèse, il convient de répondre à la question suivante: la guerre civile, ou d'une manière plus générale, les troubles sociaux ont-ils eu un impact néfaste sur la santé et l'état nutritionnel des enfants réfugiés libériens et somaliens?

La réponse à cette question nécessiterait que nous comparions deux états nutritionnels des enfants réfugiés: celui d'avant la guerre ou les troubles sociaux, et celui d'après l'exode; or les données de la première évaluation font défaut.

Néanmoins, on peut prendre le risque d'émettre certaines hypothèses : 
a). Pour les enfants réfugiés libériens en Côte-d'Ivoire et en Guinée, on peut dire, qu'étant donné que l'état nutritionnel des enfants réfugiés libériens diffère peu de celui des enfants de la population autochtone (population plus "stable" n'ayant pas connu de troubles sociaux), les troubles sociaux ont eu peu d'impact sur leur état nutritionnel. Plusieurs facteurs peuvent expliquer cette absence d'influence des troubles sociaux; parmi eux, nous pouvons citer :

- L'intégration des réfugiés libériens aux populations autochtones sans création de "camps de réfugiés": ceci était vrai en Guinée où, au début de l'exode, c'était les mêmes groupes ethniques d'accueil. C'était vrai également en Côte-d'Ivoire où il y a eu une prise en charge de la population réfugiée par la population autochtone; en effet, on a observé que "dans les villages, régnait une certaine harmonie entre communautés libériennes et communautés ivoiriennes; de nombreux Libériens se sont vu prêter des portions de terre par leurs tuteurs ivoiriens pour la culture du riz et du manioc" (Bocoum, 1991).

- La mise en oeuvre d'une stratégie efficace d'assistance aux réfugiés, tant en matière de santé qu'en ce qui concerne le maintien d'un équilibre entre les rations alimentaires octroyées aux réfugiés et l'état nutritionnel de la zone d'accueil.

b). Par contre, pour les enfants réfugiés somaliens dans l'est de l'Ethiopie, les troubles sociaux ont eu de graves effets sur leur santé et leur état nutritionnel. En outre, lorsqu'on a considéré 66663 réfugiés Somaliens, tous âges confondus, on a noté que:

- $\quad 2,1 \%$, soit 1437 réfugiés, étaient atteint de scorbut clinique (saignement des gencives et articulations douloureuses, enflées par exemple) dû au fait que les rations alimentaires ne contenaient pas souvent la dose minimale quotidienne de vitamine $\mathrm{C}$ fixé à $6 \mathrm{mg}$ et aussi à la thermostabilité de la vitamine $\mathrm{C}$;

- 60 personnes étaient atteintes d'hépatite, avec 4 décès liés à l'hépatite (sans identification du type d'hépatite A ou B).

Dans le cas présent, plusieurs facteurs explicatifs peuvent être pris en compte (WHO, 1990) :

- Difficultés d'approvisionnement des camps en eau;

- Intermittence dans la distribution des rations alimentaires (céréales, huile végétale et légumineuses);

- Irrégularité de l'approvisionnement en lentilles et huile végétale, de telle sorte que les céréales ont constitué la seule source continue de calories;

- Retard dans la distribution des rations dû aux lacunes des recensements effectués dans les camps (rations de 10 jours pour des périodes de trois à quatres semaines pour certaines familles).

\section{Conclusion}

Nous concluerons cette étude par trois observations:

D'abord, il faut noter que la définition de la "population réfugiée" doit apparaître clairement dans les études réalisées et publiées sur ce sous-groupe de population afin de permettre des comparaisons valides.

Ensuite, l'utilisation de la population de référence de NCHS ne souffre pas de limitation et ne constitue pas un obstacle à l'évaluation de l'état nutritionnel des enfants ailleurs qu'aux Etats-unis.

Enfin, il convient de noter que les réfugiés sont en général tributaires des rations alimentaires fournies par les donateurs internationaux et transportées et distribuées par les institutions des Nations-Unies et le gouvernement du pays hôte. De ce fait, les stratégies efficaces de prise en charge des réfugiés et d'amélioration de l'état nutritionnel des réfugiés, surtout des enfants, pourraient comporter (US CCCD, 1989): 
- La distribution régulière et complète de rations alimentaires comportant des aliments riches en vitamine $C$ (légumes et fruits frais par exemple) pour contenir les risques mortels de scorbut; ce qui suppose l'aplanissement des difficultés d'ordre logistique;

- L'extension des programmes d'alimentation d'appoint et de thérapeutique pour une meilleure couverture de la population d'enfants mal nourris;

- La collecte de données de morbidité plus complètes;

- et enfin le maintien de la surveillance de l'état nutritionnel des enfants.

Tableau 1 : Taille d'échantillon, types de mesures effectuées, catégories d'enfants concernées et périodes d'enquête

\begin{tabular}{|c|c|c|c|c|c|}
\hline \multicolumn{2}{|c|}{$\begin{array}{l}\text { Type de réfugiés et } \\
\text { pays d'accueil }\end{array}$} & \multirow{2}{*}{\begin{tabular}{l}
\multicolumn{1}{c}{$\begin{array}{c}\text { Taille } \\
\text { d'échantillon }\end{array}$} \\
$\begin{array}{l}495 \text { à } 511 \\
\text { (milieu rural) }\end{array}$
\end{tabular}} & \multirow{2}{*}{$\begin{array}{c}\begin{array}{c}\text { Types de } \\
\text { mesures } \\
\text { effectuées }\end{array} \\
\text { Poids/taille }\end{array}$} & \multirow{2}{*}{$\begin{array}{c}\begin{array}{c}\text { Catégories } \\
\text { d'enfants } \\
\text { (âges exacts) }\end{array} \\
6 \text { mois à } 5 \\
\text { ans }\end{array}$} & \multirow{2}{*}{$\begin{array}{l}\text { Période de } \\
\text { la collecte }\end{array}$} \\
\hline Libériens & $\begin{array}{l}\text { Côte } \\
\text { d'Ivoire }\end{array}$ & & & & \\
\hline & Guinée & 590 & Poids/taille & $\begin{array}{l}6 \text { mois à } 5 \\
\text { ans }\end{array}$ & Mai 1990 \\
\hline Somaliens & Ethiopie & $\begin{array}{l}720 \text { à } 1350 \\
\text { (passages } \\
\text { répétés) }\end{array}$ & $\begin{array}{l}\text {-Poids/taille } \\
\text {-Circonférence } \\
\text { du bras }\end{array}$ & 0 à 5 ans & $\begin{array}{l}\text { Sept. } 1988 \\
\text { à Mai } 1989\end{array}$ \\
\hline
\end{tabular}

Tableau 2 : Etat nutritionnel des enfants libériens en Côte d'Ivoire selon le type de population

\begin{tabular}{|c|c|c|c|c|c|c|c|}
\hline \multirow{3}{*}{\multicolumn{2}{|c|}{$\begin{array}{l}\text { Régions et groupes- } \\
\text { cibles }\end{array}$}} & \multirow{4}{*}{$\begin{array}{c}\text { Sujets } \\
\text { examinés }\end{array}$} & \multirow{2}{*}{\multicolumn{2}{|c|}{$\begin{array}{c}\begin{array}{c}\text { Bon état } \\
\text { nutritionnel }\end{array} \\
>-2 \mathrm{ET}\end{array}$}} & \multirow{2}{*}{\multicolumn{2}{|c|}{$\begin{array}{c}\begin{array}{c}\text { Mauvais état } \\
\text { nutritionnel }\end{array} \\
=<-2 \text { ET }\end{array}$}} & \multirow{3}{*}{$\begin{array}{c}\begin{array}{c}\% \text { des mal- } \\
\text { nourris }\end{array} \\
\mathrm{PT}<80 \% \\
\text { de la } \\
\text { médiane }\end{array}$} \\
\hline & & & & & & & \\
\hline & & & Effectif & $\%$ & Effectif & $\%\left(^{*}\right)$ & \\
\hline DANANE & Libériens & & 490 & 96.2 & 19 & $\begin{array}{l}3.8(1.5- \\
\quad 6.1)\end{array}$ & 2.9 \\
\hline & Ivoiriens & 504 & 477 & 94.2 & 27 & $\begin{array}{l}5.4(2.6- \\
8.2)\end{array}$ & 3.2 \\
\hline GUIBGLO & Libériens & 511 & 489 & 95.7 & 22 & $\begin{array}{l}4.3(1.8- \\
\quad 6.8)\end{array}$ & 4.3 \\
\hline & Ivoiriens & 495 & 479 & 96.8 & 16 & $\begin{array}{c}3.2(1.0- \\
5.4)\end{array}$ & 3.2 \\
\hline
\end{tabular}

Source : SORO, 1993, p.7

* Intervalle de confiance pour un risque d'erreur de $5 \%$ 
Tableau 3 : Pourcentage d'enfants libériens et guinéens souffrant de malnutrition en Guinée, mai 1990

\begin{tabular}{||c|c||c|c||}
\hline \multicolumn{1}{|c|}{ à la taille } & Guinéens & Libériens & Total \\
\hline \hline & $(\mathrm{n}=314)$ & $(\mathrm{n}=590)$ & $(\mathrm{n}=904)$ \\
\hline \hline $\begin{array}{l}\text { Médiane de référence } \\
(\mathrm{a})\end{array}$ & 0.3 & 0.5 & 0.4 \\
$<70 \%$ & 5.4 & 5.3 & 5.3 \\
$<80 \%$ & 6.7 & 11.4 & 9.7 \\
$80-85 \%$ & 7.3 & 8.4 & 8.0 \\
\hline \hline$<-2$ ET & 7.3 \\
\hline \hline
\end{tabular}

Note : (a) Malnutrition aiguë : $<80 \%$ de la médiane du poids par rapport à la taille ou $<-2$ ET Malnutrition sévère ou grave: $<70 \%$ de la médiane du poids par rapport à la taille.

Source : WHO, 1990.

Tableau 4 : Etat nutritionnel des enfants réfugiés somaliens de moins de 5 ans en Ethiopie, septembre 1988 - mai 1989

\begin{tabular}{|c||c||c||c||c|c||}
\hline Camp & Période & $\begin{array}{c}\text { Nombre } \\
\text { de } \\
\text { passage }\end{array}$ & $\begin{array}{c}\text { Nombre } \\
\text { d'enfants } \\
\text { examinés }\end{array}$ & $\begin{array}{c}\text { Pourcentage } \\
\text { dont le poids } \\
\text { pour la taille } \\
\text { est }<80 \%\end{array}$ & $\begin{array}{c}\text { Pourcentage dont } \\
\text { le poids pour la } \\
\text { taille est < } \\
\text { (malnutrition } \\
\text { grave) }\end{array}$ \\
\hline \hline Hartisheik & $\begin{array}{c}\text { Sept. } \\
1988 \\
\text { à Mai } \\
1989\end{array}$ & 5 & $\begin{array}{c}1080 \text { à } \\
1350\end{array}$ & 16.9 à 26.4 & 1.8 à 4.3 \\
\hline \hline Harshin & $\begin{array}{c}\text { Janvier à̀ } \\
\text { Mai } \\
1989\end{array}$ & 3 & 690 à & 12.5 à 29.5 & 1.8 à 4.9 \\
\hline
\end{tabular}

Source : WHO, 1990, p.94.

\section{Bibliographie}

- Bocoum M.D. (1991) Identification des besoins des réfugiés Libériens: femmes, chefs de famille et mineurs non accompagnés et propositions de programmes d'activités, UNHCR, Bureau du chargé de mission pour la Côte-d'Ivoire, Abidjan, décembre 1991, 30 p. + annexes.

- Graitcer P. L., et Gentry M. (1981), 'Measuring children: one reference for all', Lancet, 2: 297299.

- Habicht J. P. et al.(1974) `Height and Weight standards for pre-school children. How relevant are ethnic differences in growth potential ?', Lancet, 1: 611-615 (cité par WHO, 1986). 
- Soro B.N.(1993) Evaluation de l'état nutritionnel des enfants de 6 à 59 mois dans la zone d'accueil des réfugiés Libériens en Côte-d'Ivoire (étude réalisée en milieu rural2, HCR/Délégation pour la Côte-d'Ivoire, Abidjan, janvier 1993, 16 p.

- Togo, Direction de la Statistique (1989) "Anthropométrie de lapopulation urbaine", Télégramme EBC, $\mathrm{n}^{\circ}$ 4, Lomé, novembre 1989, pp. 1-18.

- UNHCR (1993) Refugee enumeration and statistics: A review of UNHCR policies and practices. Part one: Mass determination programmes, UNHCR, Central Evaluation Section, EVAL/STAT/13, March 1993, pp. 1-11, 21-32.

- US CCCD (1989) 'Morbidity and mortality', Weekly Report, 38, n²6, US Centers for Disease Control.

- WHO (1985) `Energy and protein requirements: report of a joint FAO/WHO/ONU Expert consultation', Technical Report Series, $n^{\circ} 724$, OMS, (cité par WHO, 1986).

- WHO (1986) 'Use and interpretation of anthropometric indicators of nutritional status', Bulletin of the World Health Organisation, 64(6): 929-941.

- WHO (1990) "Etat nutritionnel des réfugiés Somaliens dans l'est de l'Ethiopie", Septembre 1988 - Mai 1989, Relevé épidémiologique Hebdomadaire, 1990, n¹3, pp. 93-95.

- WHO (1991) "Etat de santé et état nutritionnel des réfugiés du Libéria", Relevé épidémiologique Hebdomadaire, 1991, n 16, pp. 112-113. 